\title{
Analysis of Posterior Probability Uncertainty for Classification of Hyperspectral Images by SupportVector Machines
}

\author{
Xiaoxia SUN ${ }^{12}$, Liwei $\mathrm{LI}^{1 *}$, Bing Zhang ${ }^{1}$, Ling YANG $^{2}$ \\ 1. Lab of Digital Earth, Chinese Academy of Sciences, Beijing, China \\ 2. College of Environment and Planning, Henan University, Kaifeng, China \\ *: Correspondent Author Email: 1wl@ceode.ac.cn
}

\begin{abstract}
This paper analyzes the uncertainty of classification posterior probability of support vector machine (SVM) using urban hyperspectral images. The hyperspectral images in Zhangye are selected as the study zone, and the sample parameter data were acquired based on the high resolution images and the ground survey information, the images were classified with parameter-optimized SVM to obtain the posterior probability graph for each class, and the posterior probability graphs were truncated using the threshold values of $0.2,0.4,0.6$, 0.8 and 0.9 for analysis of the accuracy change of ground object classification at different probabilities. The results show that with the increase of truncation probability, the user accuracy in the classification results increases continuously, while the producer accuracy shows a declining tendency, and the overall classification accuracy also shows a declining tendency. The analysis of the posterior probability distribution of various types of ground objects shows that it is difficult to distinguish the posterior probability of some mixed ground objects. The untrained water body targets can be easily distinguished by the truncation probability, but the posterior probabilities of untrained red materials and white materials are mixed together. This shows that there exist some conditions in which the posterior probability of optimized SVM can not directly and effectively indicate the distinction of ground objects. The posterior probability should be used optionally, and at the same time, it is necessary to construct a more robust calculation method for the posterior probability.
\end{abstract}

IndexTerms-SVM, hyperspectral, posterior probability, classification, uncertainty.

\section{INTRODUCTION}

Supervised classification algorithm requiresthat training sample data can represent the statistical characteristics of the data to be classified [3], [4]. In practical applications, as some non-interest category is deliberately excluded in the training phase or some unknown categoryis ignored unconsciously, the training samples can not fully represent all ground objects [2]. In such case, the classification results and accuracy can not fully represent the classification results and accuracy of the whole image [1]. Classification posterior probability can indicate the uncertainty of classification results [8]. The analysis of classification posterior probability is able to guide the acquisition of the information on the unclassified ground objects to improve the accuracy of classification results. It has practical significance in remote sensing image classification applications [5].
In recent years, support vector machine (SVM), as a supervised classification algorithm, shows very good performancesin remote sensing data classification andis especially widely used in hyperspectral data classification [6]. The statistic analysis based on two-two classification results can produce the posterior probability of classification by SVMs[7]. Presently, the research interests are mainly focusing on high-precision classification of ground objects by SVM when there are sufficient samples, while fewer efforts are made in analysis of the posterior probability of classification by SVMs.In this paper, we analyze the posterior probability uncertainty of classification by SVM based on urban hyperspectral images to guide the application of classification algorithms using SVMs on condition that there are incomplete samples.

\section{DATA AND EXPERIMENTAL DESIGN}

A. Data

The airbornehyperspectral data on the areas surroundingHexi University, Zhangye City, Gansu province, are selected as thestudy zone. It has a size of $1000 * 1000$ pixels, a band number of 48 , a spectrum range of $380-1055 \mathrm{~nm}$ anda spatial resolution of $1 \mathrm{~m}$ (as shown in Fig. 1), in which $\mathrm{R}, \mathrm{G}$ and $\mathrm{B}$ are displayed with 19 bands, 13 bands and 6 bands respectively.

Eight classes were selected based on the high resolution images and ground survey information respectively, i.e., artificial grasses, red materials and water bodies, and a certain number of samples are selected for each class as the test samples. $10 \%$ of the five classes of gray materials, white materials, blue roofs, bare lands andvegetationswere randomly selected as the samples for supervising the training phase in classification, and artificial grasses, red materials and water bodies are combined into unclass for testing the stability of probability graph. Therefore, the training samples for the test include 5 classes, and the test samples include 6 classes, covering the category of unclass. The spatialdistribution of the test and training samplesare identified in Fig. 1.

B. Test design

Classify the study area by SVM using the training samples and optimize the parameters using the grid search method. The minimum and maximum values of the Kernel parameter are 0.1 and 1000 respectively, the search multiple was 10 , the minimum and maximum values of the 
Table 1 Number of training and testsamples in different classes

\begin{tabular}{ccccccc}
\hline Class & Gray materials & White materials & Blue roofs & Bare lands & Vegetations & Unclass \\
\hline Train & 384 & 235 & 129 & 152 & 293 & 0 \\
Test & 3835 & 2345 & 1291 & 1519 & 2926 & 4499 \\
\hline
\end{tabular}

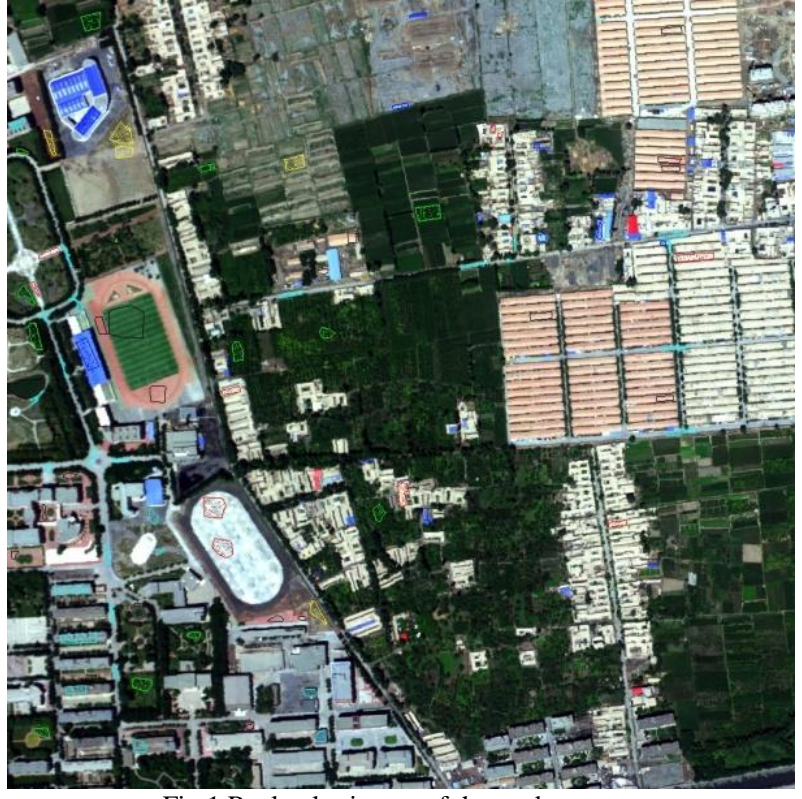

Fig.1 Real color image of the study zone

value of the Kernel parameter is 10, and the value of Regularization parameter is 100 , and the Cross Validation method is used to assess the training results.

To illustrate the guidance role of probability graph in classification results, select the six threshold values of $0,0.2$, 0.4, 0.6, 0.8 and 0.9 to carry out threshold value truncation for the probability graph. Calculate the confusion matrix of the graph for each class after different threshold values are truncated using the same test samples, and then calculate the producer and user accuracy of the graph for each class and the overall accuracy of the classification results.

\section{III.RESULT ANALYSIS}

The classification results with threshold values of $0,0.4,0.8$ and 0.9 are showed in Fig. 2-5.The comparison of the graphs of the classification results for each threshold value show thatthere are more and more unclassified black zones that are distributed increasingly wide ranges. When the threshold value is 0 ,it is the graph of the classification result of the five classes; when the threshold value is 0.2 ,it is impossible to distinguish the unclass; when the threshold value is 0.4 , the water bodies are clear distinguished; when the threshold values is 0.6 and 0.8 , the results are basically the same (the water bodies and a small amount of red materialsareclearly distinguished and the shadow of part of the roofs and the boundary area of vegetations and bare lands are distinguished); when the threshold value is 0.9 , the water bodies, the roads in red materials, a small part of bright red roofs and part of artificial grassesare distinguished. But the red roofs and artificial grass are still can not be distinguished.

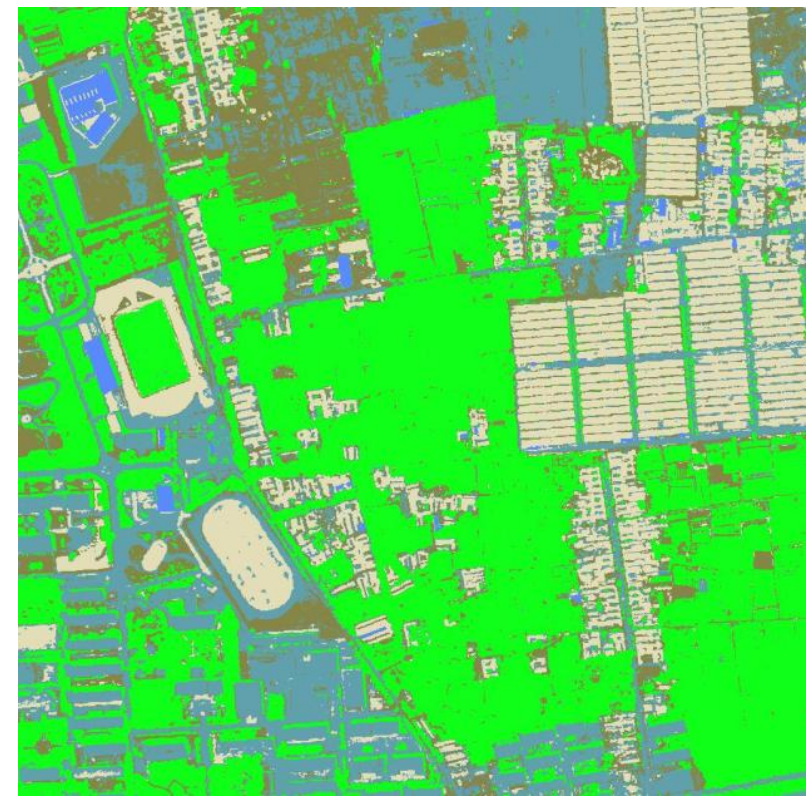

Fig. 2 The classification result with threshold value of 0 (black indicate unclassified zones)

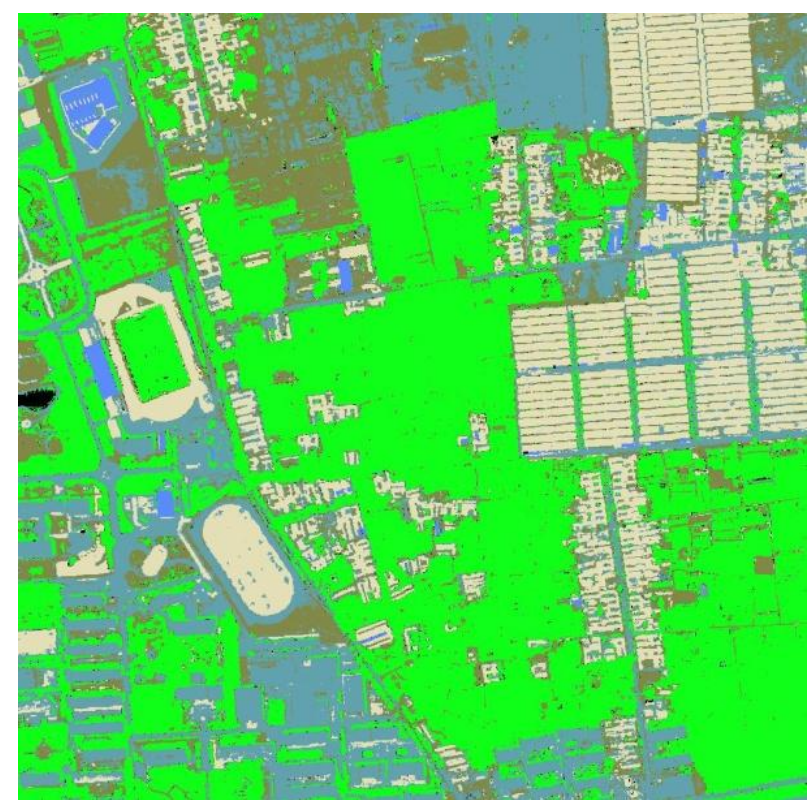

Fig. 3 The classification result with threshold value of 0.4 (black indicates unclassified zones) 


\begin{tabular}{|c|c|c|c|c|c|c|c|c|c|c|}
\hline \multirow{2}{*}{$\begin{array}{c}\text { Threshold } \\
\text { Class }\end{array}$} & \multicolumn{2}{|c|}{$\mathbf{0}$} & \multicolumn{2}{|c|}{0.4} & \multicolumn{2}{|c|}{0.6} & \multicolumn{2}{|c|}{0.8} & \multicolumn{2}{|c|}{0.9} \\
\hline & $\underline{\text { User }}$ & Prod & $\underline{\text { User }}$ & Prod & $\underline{\text { User }}$ & Prod & $\underline{\text { User }}$ & Prod & $\underline{\text { User }}$ & Prod \\
\hline Gray materials & 95.74 & 97.84 & 95.89 & 97.84 & $\underline{97.16}$ & 97.29 & 97.16 & 97.3 & $\underline{99.19}$ & 89.67 \\
\hline White materials & $\underline{58.08}$ & 97.19 & $\underline{57.98}$ & 96.43 & $\underline{58.2}$ & 95.78 & $\underline{58.2}$ & 95.8 & $\underline{61.75}$ & 90.19 \\
\hline Blue roofs & $\underline{100}$ & 99.54 & $\underline{100}$ & 99.54 & $\underline{100}$ & 99.46 & $\underline{100}$ & 99.5 & $\underline{100}$ & 99.38 \\
\hline Bare lands & $\underline{75.01}$ & 98.42 & $\underline{75.09}$ & 98.42 & $\underline{76.31}$ & 97.76 & $\underline{76.31}$ & 97.8 & $\underline{78.66}$ & 88.08 \\
\hline Vegetations & $\underline{55.21}$ & 99.9 & $\underline{64.34}$ & 99.9 & $\underline{65.71}$ & 99.86 & $\underline{65.71}$ & 99.9 & $\underline{71.91}$ & 99.49 \\
\hline Unclassified & $\underline{0}$ & 0 & $\underline{98.83}$ & 16.87 & $\underline{88.35}$ & 20.4 & $\underline{88.35}$ & 20.4 & $\underline{68}$ & 37.65 \\
\hline
\end{tabular}

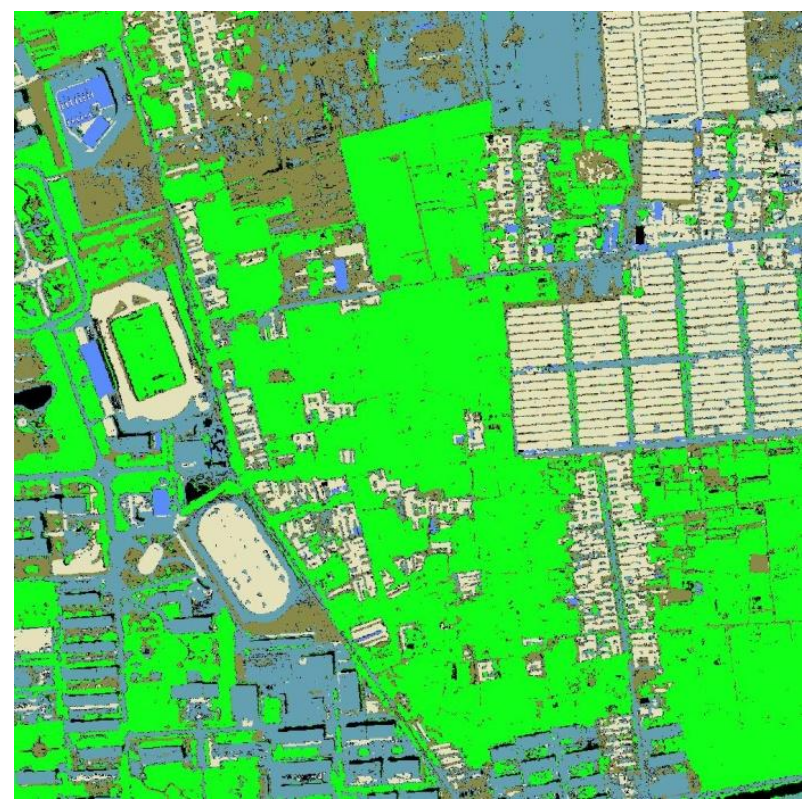

Fig. 4 The classification result with threshold value of0.8 (black indicates unclassified zones)

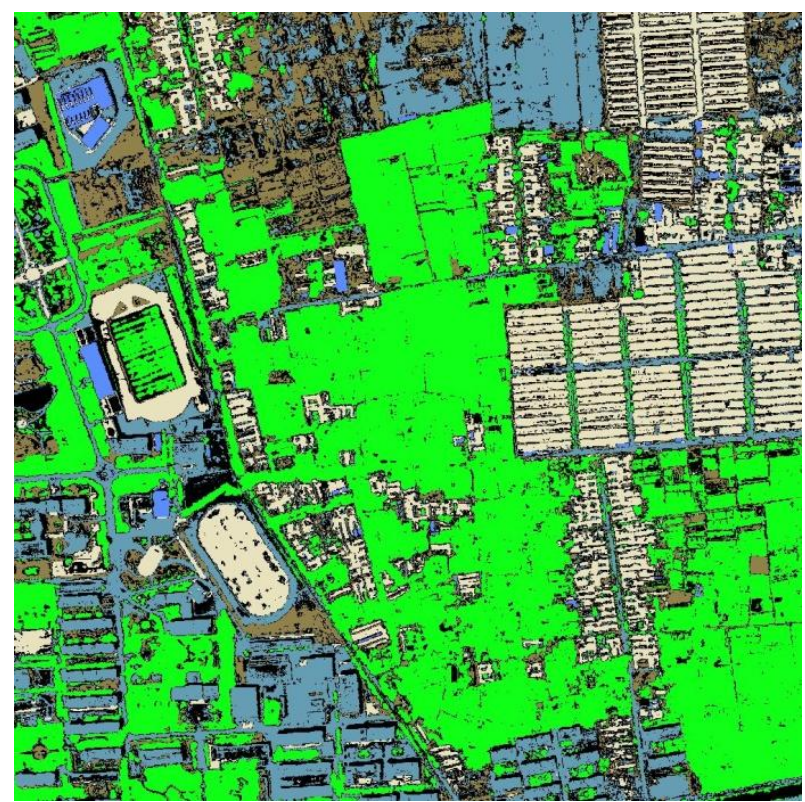

Fig. 5 The classification result with threshold value of0.9 (black indicates unclassified zones)
The statistics for the accuracy of the classification results when different threshold values are truncated is shown Table 2.

From theproducer perspective, with the increase of the truncated threshold values, the accuracy of almost all interest classes decreases, but the uncertain zonesobtained areincreasingly larger, the number of unclassified zones is increasing, and the calculationaccuracy of unclassified zones is also increasingly higher. From the user perspective, with the increase of the truncated threshold values, the accuracy of almost all interest classesis improved. Among them, the accuracy of blue roofs and gray materialsalways maintains highest, followed by the accuracy of bare lands and vegetations, and the accuracy of white materials is lowest.

As shown by the classification results and the analysis of the spectral data of the image samples, somebare soil among crops are mixed with a small amount of weeds, making it difficult to distinguish spectrally the boundary area of bare lands and vegetations, some bare soil among buildings are mixed with impurities and stone, causing the confusion between it and other materials, and it is difficult to distinguishthe posterior probability of the confusing ground objects. The untrained water body targets can be easily distinguished by the truncation probability. However, although there are noticeable spectral difference between white materials and red materials, their posterior probabilities are not easily distinguished. This shows that, the posterior probability of optimizedSVMcan not effectively indicatethe distinguishability between red materials and white materials, so it is necessary to further study the causes for the abnormalities of the posteriori probability of combine with probability of classification by SVMsin combination with the spectral analysis of ground objects and the posterior probabilitygenerating mechanism so as to construct the more robust calculation method for posterior probability.

\section{IV.CONCLUSION}

This paper analyzes the uncertaintyof classification posterior probability of support vector machine (SVM)using urban hyperspectral images. The results show that the posterior probability can be used to detect the untrained classesin the classification results of SVM and with the increase of the threshold values, more classes are detected, but there are some circumstances in which the posterior probability of optimized SVM cannot directly and effectively indicate the distinguishability of ground objects. Therefore, the posterior probability should be used optionally, and at the same time, it 
is necessary to construct a more robust calculation method for the posterior probability.

\section{ACKNOWLEDGMENT}

This research is funded by National 863 programs (No. 2009AA12Z102). Special thanks to colleaguesin the Heihe experiment for providing the data.

\section{REFERENCES}

[1] Foody, G.M., "Hard and soft classifications by a neural network with a non-exhaustively defined set of classes. International Journal of Remote Sensing," Vol. 23, pp. 3863-3864, November 2002.

[2] Jeon, B. and Landgrebe, D.A., "Partially supervised classification using weighted unsupervised clustering," IEEE Transactions on Geoscience and Remote Sensing, Vol. 37, pp. 1073-1079, March 1999.

[3] Leckie, D.G., "Synergism of synthetic aperture radar and visible/infrared data for forest type discrimination," Photogrammetric Engineering and Remote Sensing, Vol. 56, pp. 1237-1246, 1990.
[4] Lewis, H.G. and Brown, M., "A generalized confusion matrix for assessing area estimates from remotely-sensed data," International Journal of Remote Sensing, Vol. 22, pp. 32233235, November 2001.

[5] Li, L., Ma, J. and Wen, "Comparison of local transfer function classifier and radial basis function nueral network with and without an exhaustively defined set of classes," International Journal of Remote Sensing, Vol.30, pp. 85-96, January 2009.

[6] Shao Yang, Lunetta, Ross S, "Comparison of support vector machine, neural network, and CART algorithms for the landcover classification using limited training data points," ISPRS Journal of Photogrammetry and Remote Sensing, Vol. 70, pp. 78-87, June 2012.

[7] Ting-Fan Wu, Chih-Jen Lin, Ruby C. Weng, "Probability estimates for multi-class classification by pairwise coupling," Journal of Machine Learning Research, Vol. 5, pp. 975-1005, August 2004.

[8] John A. Richards, XiupingJia, "A dempster-shafer relaxation approach to context classification," IEEE Transaction on Geoscience and Remote Sensing, Vol. 45, pp. 1422-1431, May 2007. 